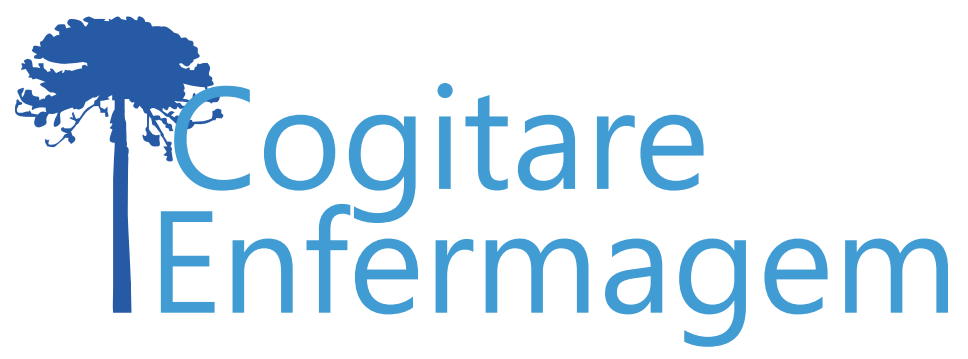

REVISÃO

\title{
ESTRATÉGIAS PARA A REDUÇÃO DE ERROS DE MEDICAÇÃO DURANTE A HOSPITALIZAÇÃO: REVISÃO INTEGRATIVA
}

\author{
STRATEGIES FOR REDUCING MEDICATION ERRORS DURING \\ HOSPITALIZATION: INTEGRATIVE REVIEW
}

\author{
Claudia Regina de Barros Costa ${ }^{1}$ (c) \\ Sara Soares dos Santos ${ }^{2}$ (1) \\ Simone de Godoy ${ }^{2}$ (1) \\ Leila Maria Marchi Alves² \\ Ítalo Rodolfo Silva ${ }^{3}$ (1) \\ Isabel Amélia Costa Mendes ${ }^{2}$ (10
}

\begin{abstract}
Objective: to synthesize the knowledge related to strategies to reduce medication errors during the hospitalization of adult patients. Method: integrative literature review carried out in October 2020 through the search for publications in electronic databases: Web of Science, Pubmed, Cumulative Index of Nursing and Allied Health Literature, Latin American and Caribbean literature in Health Sciences and Google Scholar. Results: sample composed of 12 studies, whose strategies for the reduction of medication errors were presented according to the categories: involvement of the clinical pharmacist in clinical activities, implementation of information technologies, educational strategies mediated by simulation and games, and workload reduction. Conclusion: associated with technologies, the involvement of the clinical pharmacist in the medical and nursing team will result in improvements in the reduction of adverse medication events and in the quality of care provided to the patient.
\end{abstract}

DESCRIPTORS: Medication errors; Patient Safety; Hospital Medication Systems; Safety Management; Nursing Care.

COMO REFERENCIAR ESTE ARTIGO:

Costa CR de B, Santos SS dos, Godoy S de, Alves LMM, Silva IR, Mendes IAC. Estratégias para a redução de erros de medicação durante a hospitalização: revisão integrativa. Cogit. Enferm. [Internet]. 2021 [acesso em "colocar data de acesso, dia, mês abreviado e ano"]; 26. Disponível em: http://dx.doi.org/10.5380/ce.v26i0.79446. 
Organizações internacionais de saúde consideram a segurança do paciente como indicador prioritário da qualidade do cuidado. Esforços em todo o mundo são direcionados para reduzir e controlar os riscos originados de incidentes de segurança(1). Dentre os eventos que causam consequências danosas e evitáveis, estão os erros de medicação. A identificação e o relato de erros de medicação são essenciais para se resguardar a segurança do paciente ${ }^{(2)}$, uma vez que podem ocorrer em todas as fases do processo de uso de medicamentos: na prescrição, dispensação e administração, além das situações de conciliação medicamentosa, e no monitoramento inadequado do paciente ${ }^{(3)}$.

Os erros de medicação são evitáveis e considerados uma das importantes causas de agravos à saúde em todo mundo, por resultarem em elevadas taxas de mortalidade, prolongarem o período de hospitalização, afetarem o tratamento, além de aumentarem as despesas da assistência em saúde(4). Segundo análise da Organização Mundial da Saúde (OMS), divulgada em 2017, os custos mundiais associados a erros de medicação são de aproximadamente US\$ 42 bilhões por ano ${ }^{(5-6)}$.

Esses dados sobre os custos dos erros de medicação geraram iniciativas globais para uma meta de redução de $50 \%$ dos danos graves e evitáveis relacionados à medicação, de modo que o Global Patient Safety Challenge, da OMS, elegeu a "Medicação Sem Danos" como seu terceiro Desafio Global, visando expor e discutir as fragilidades nos sistemas de saúde e estabelecer estratégias de prevenção de tais ocorrências ${ }^{(5)}$. Os fatores desencadeantes dos erros de medicação são múltiplos e parte deles é originada por falhas de comunicação entre os profissionais de saúde e problemas relacionados à gestão e organização (7). No entanto, os fatores psicossociais desempenham um papel crucial no surgimento desses erros ${ }^{(8)}$.

É reconhecido que erros de medicação podem resultar em danos temporários ou permanentes ao paciente. Entretanto, os resultados decorrentes desses erros podem ser minimizados ou revertidos caso o erro seja identificado precocemente ${ }^{(9)}$. À vista disso, o National Coordinating Council for Medication Error Reporting and Prevention (NCC-MERP) dos Estados Unidos da América recomenda que as organizações de saúde desenvolvam sistemas e processos para coletar informações necessárias para análise de relatos de erros de medicação o mais rápido possível, a partir da ocorrência( ${ }^{(9)}$.

Tão importante quanto agir precocemente a fim de reparar prejuízos causados pelos erros de medicação, é implementar estratégias para identificar e minimizar os fatores predisponentes a riscos. Várias estratégias para prevenir esses erros têm sido estudadas e utilizadas em todas as etapas da cadeia de uso de medicamentos, como a utilização de tecnologias da informação em saúde, as quais reduzem significativamente os riscos ${ }^{(10)}$.

Outra estratégia amplamente difundida entre a equipe de enfermagem é a utilização dos "cinco certos na administração de medicamentos", atualizados para "nove certos na administração de medicamentos" (paciente certo, medicamento certo, via certa, hora certa, dose certa, registro certo, ação certa, forma certa e resposta certa) $)^{(11-12)}$. Apesar de não garantirem completamente a prevenção de erros, sua adoção sistemática pode limitar significativamente parte dos eventos ${ }^{(12)}$.

Diante do exposto, conhecer estratégias de redução de erros de medicação é essencial e pode fornecer informações úteis e necessárias para que hospitais públicos e privados superem esses problemas, adotando práticas eficazes que minimizem os danos aos pacientes e ao sistema. Nesse contexto, este estudo teve como objetivo sintetizar o conhecimento relacionado às estratégias para redução de erros de medicação durante a hospitalização de pacientes adultos. 
Estudo de revisão integrativa da literatura, desenvolvido com a finalidade de reunir e sintetizar resultados de pesquisas, de forma sistemática, independente do delineamento utilizado. Foi desenvolvido a partir das etapas de definição da questão norteadora, busca de estudos primários nas bases de dados, extração de dados dos estudos, avaliação dos estudos primários selecionados, análise e síntese dos estudos incluídos, e apresentação da síntese do conhecimento(13).

A questão norteadora da pesquisa foi formulada utilizando-se a estratégia PICO ${ }^{(14)}$, cujo acrônimo representa: P (população) - paciente adulto; I (intervenção) - estratégias de prevenção de erros de medicação; C (comparação) - não se aplicou; e O (desfecho) - redução de erros de medicação. Assim, obteve-se a seguinte pergunta: Quais são as estratégias utilizadas para redução de erros de medicação durante a hospitalização do paciente adulto?

Os critérios de inclusão adotados foram: estudos que apresentassem alguma estratégia para redução de erros de medicação durante a hospitalização de pacientes adultos, publicados nos idiomas português, inglês e espanhol, em um recorte temporal entre janeiro de 2015 a outubro de 2020, a fim de discutir as evidências científicas dos últimos cinco anos. Foram excluídos os artigos que não responderam à questão de pesquisa, os realizados com pacientes pediátricos, teses, dissertações, editoriais, artigos de revisão, opinião, reflexão ou comentário, carta ao editor, resumos expandidos e em anais de eventos científicos e livros.

Os descritores Medication errors, Hospitalization, Patient safety e Medication errors reduction foram utilizados no idioma inglês, e combinados aos operadores booleanos OR e AND nas bases de dados.

As buscas foram realizadas em outubro de 2020, de maneira independente por dois pesquisadores, nas bases de dados eletrônicas: Web of Science, Pubmed, Cumulative Index of Nursing and Allied Health Literature (CINAHL), Literatura Latino-Americana e do Caribe em Ciências da Saúde (LILACS) e Google Scholar. Foram encontrados 2.551 estudos, sendo sete na Web of Science, 2.535 na PubMed, seis na CINAHL, três na LILACS e nenhum estudo no Google Scholar.

Após a busca, os dados foram exportados para o gerenciador de referências Web EndNote para a exclusão de duplicatas. Foram identificados 12 registros duplicados. Posteriormente foi realizada a leitura de títulos e resumos por dois revisores independentes, que excluíram 2.516 registros que não responderam à questão norteadora, totalizando 23 artigos para leitura do texto na íntegra. Nesta etapa, houve divergência entre dois revisores em três artigos, sendo necessária a colaboração de um terceiro revisor para a decisão final, totalizando uma amostra final de 12 artigos incluídos na revisão (Figura 1). 


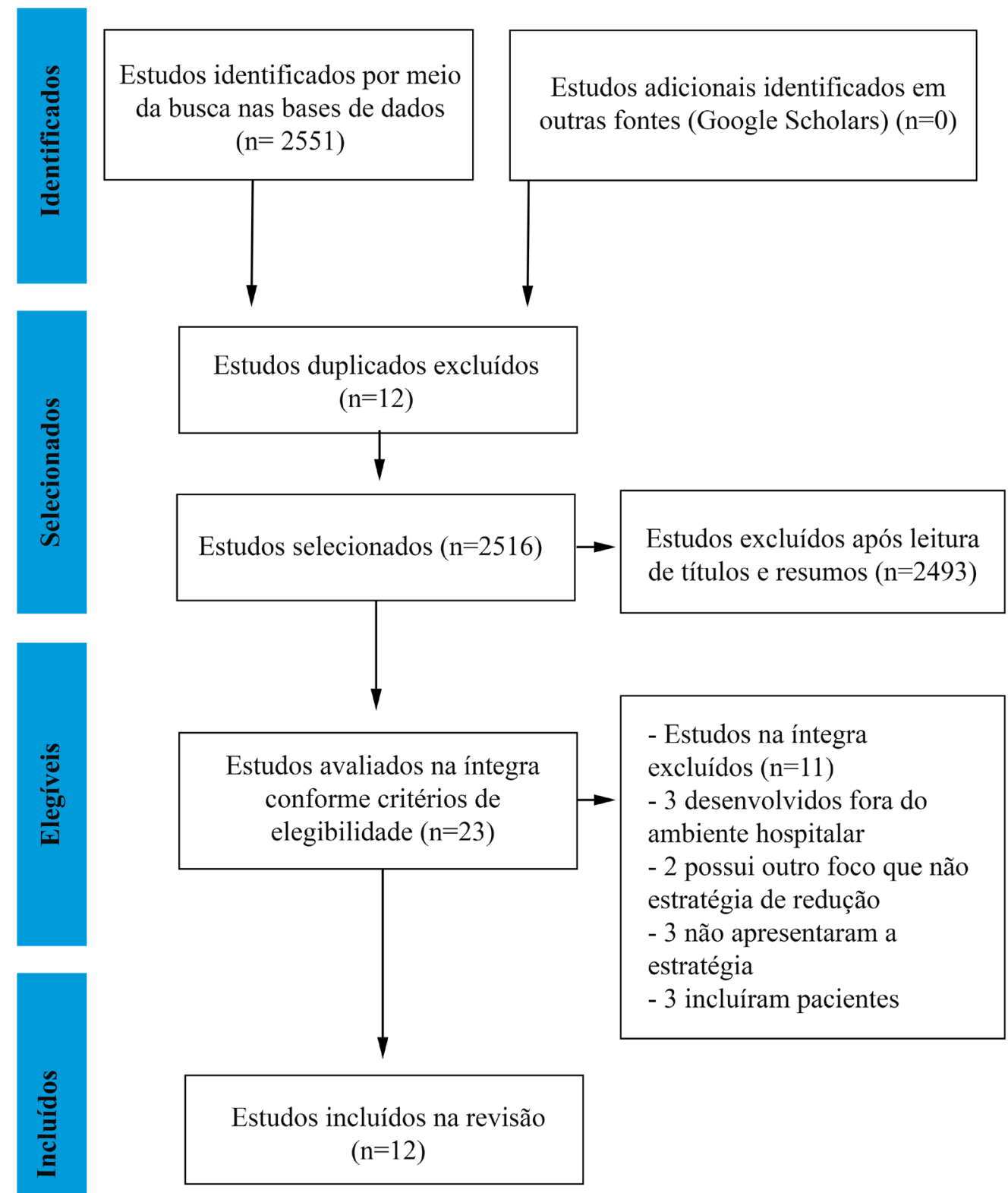

Figura 1 - Fluxograma de identificação dos estudos primários incluídos na revisão. Ribeirão Preto, SP, Brasil, 2020

Fonte: Autores (2020).

A extração dos dados para síntese qualitativa foi realizada utilizando-se instrumento validado(15) e adaptado para esta pesquisa, que contemplou: identificação do artigo, autoria, ano, idioma, características metodológicas, tratamento dos dados, intervençỗes e resultados relacionados à redução de erros de medicação durante a hospitalização do paciente adulto. Para cada estudo analisado, elaborou-se um quadro com o ano de publicação, o país, o local de pesquisa, o periódico e área de especialidade e o nível de evidência. Para a classificação do nível de evidência, foi utilizada a hierarquia de evidência em sete níveis ${ }^{(16)}$. A síntese das evidências foi organizada de maneira descritiva. 
A revisão integrativa aqui apresentada resultou de 12 artigos que atenderam aos critérios de inclusão previamente estabelecidos, sendo 10 publicados em inglês e dois publicados em português (Quadro 1).

Quadro 1 - Artigos incluídos na revisão integrativa, segundo periódico, país de realização, ano de publicação, local da pesquisa, área de especialidade do periódico e nível de evidência. Ribeirão Preto, SP, Brasil, 2020

\begin{tabular}{|c|c|c|}
\hline Periódico/país de realização/ano & Local da pesquisa & $\begin{array}{l}\text { Especialidade do periódico } \\
\text { / nível de evidência }\end{array}$ \\
\hline $\begin{array}{l}\text { Therapeutics and Clinical Risk } \\
\text { Management, China, 2015(17) }\end{array}$ & Hospital de ensino & Interdisciplinar (VI) \\
\hline PloS One, França, 2015(18) & $\begin{array}{l}\text { Hospital de ensino, unidade } \\
\text { cirúrgica ortopédica }\end{array}$ & Interdisciplinar (VI) \\
\hline Pharmacy Practice, EUA, 2015(19) & $\begin{array}{l}\text { Hospital comunitário, com } \\
\text { fins lucrativos, filiado ao } \\
\text { sistema de saúde do Hospital } \\
\text { Corporation of America (HCA) }\end{array}$ & Farmácia (IV) \\
\hline $\begin{array}{c}\text { Revista Latino-Americana de } \\
\text { Enfermagem (RLAE), Brasil, 2016(20) }\end{array}$ & Hospital público & Enfermagem (VI) \\
\hline $\begin{array}{l}\text { European Journal of Hospital Pharmacy } \\
(\text { EJHP), Dinamarca, 2016(21) }\end{array}$ & $\begin{array}{l}\text { Hospital de ensino, } \\
\text { hematológico }\end{array}$ & Farmácia hospitalar (IV) \\
\hline $\begin{array}{l}\text { Journal of Research in Pharmacy Practice } \\
\text { (JRPP), Irã, 2017(22) }\end{array}$ & Hospital de ensino & Farmácia (VI) \\
\hline Epidemiology and Health, Irã, 2017(23) & Hospital de ensino & $\begin{array}{l}\text { Epidemiologia e Saúde } \\
\text { Pública (VI) }\end{array}$ \\
\hline PLoS One, França, $2017^{(24)}$ & Hospital de ensino & Interdisciplinar (IV) \\
\hline $\begin{array}{c}\text { BMJ Quality Improvement Reports, } \\
\text { Holanda, 2017(25) }\end{array}$ & Hospital de ensino & Saúde (III) \\
\hline Hospital Pharmacy, EUA, $2018^{(26)}$ & Hospital de ensino & Farmácia (IV) \\
\hline $\begin{array}{c}\text { Therapeutic Advances in Drug Safety, } \\
\text { Austrália, 2019(27) }\end{array}$ & Hospital de ensino & Interdisciplinar (III) \\
\hline Einstein (São Paulo), Brasil, 2019(28) & Hospital de ensino & Saúde (VI) \\
\hline
\end{tabular}

Fonte: Autores (2020).

Com relação ao local onde os estudos foram desenvolvidos, verificou-se que em 10 artigos os dados são provenientes de hospitais de ensino filiados a universidades, um de hospital público e um de hospital comunitário com fins lucrativos. Constatou-se, também, que 10 pesquisas foram realizadas em uma única instituição e duas foram multicêntricas.

Considerando o ano de publicação, verifica-se que três artigos foram publicados em 2015, dois em 2016, quatro em 2017, um em 2018 e dois em 2019.

Quanto à especialidade do periódico em que os artigos foram publicados, quatro são de revistas interdisciplinares, três da farmácia, dois de saúde em geral, um da enfermagem, um de farmácia hospitalar e um da área de saúde pública e epidemiologia. Em termos dos níveis de evidência, dois artigos foram classificados em nível III, quatro em nível IV e seis em nível VI. 
Com relação ao detalhamento metodológico, dos artigos selecionados somente um teve abordagem qualitativa com análise de conteúdo de Graneheim; os demais utilizaram abordagem quantitativa com delineamento transversal (um), observacional antes e depois (dois), coorte prospectivo (dois), coorte retrospectivo (dois), quase experimental (dois) e intervenção (dois).

As estratégias de redução de erros de medicação identificadas compreenderam a incorporação de sistemas eletrônicos desde a prescrição até a administração de medicamentos, a colaboração do farmacêutico e familiares no processo de administração de medicamentos e reconçiliação medicamentosa, a educação permanente da equipe de saúde, bem como o uso de método baseado no esforço colaborativo da equipe para melhor desempenho no processo de administração de medicamentos (Quadro 2).

Quadro 2 - Artigos incluídos na revisão integrativa de acordo com o título e as estratégias de redução de erros de medicação encontradas, Ribeirão Preto, SP, Brasil, 2020 (continua)

\section{Título do artigo}

Quality improvements in decreasing medication administration errors made by nursing staff in an academic medical center hospital: a trend analysis during the journey to Joint Commission International accreditation and in the post-accreditation era ${ }^{(17)}$

Causes of Medication Errors in Intensive Care Units from the Perspective of Healthcare Professionals ${ }^{(22)}$

\section{Estratégias de redução de erros de medicação}

Programa de intervenção de três anos e meio com foco em erros de medicação visando o credenciamento da instituição na Joint Commission International (JCl)

Entrada computadorizada do pedido de prescrição; formato idêntico para a redação da prescrição; educação permanente a equipe de saúde; recurso humano eficaz no atendimento; colaboração do farmacêutico; envolvimento de familiares/cuidadores no processo de reconciliação de medicamentos na internação.

Medication errors among nurses in teaching hospitals in the west of Iran: what we need to know about prevalence, types, and barriers to reporting ${ }^{(23)}$

An Observational Study of the Impact of a Computerized Physician Order Entry System on the Rate of Medication Errors in an Orthopaedic Surgery Unit ${ }^{(18)}$

Improving medication safety: Development and impact of a multivariate model-based strategy to target high-risk patients ${ }^{(24)}$

The Role of Computerized Clinical Decision Support in Reducing Inappropriate Medication Administration During Epidural Therapy ${ }^{(26)}$

An automated medication system reduces errors in the medication administration process: results from a Danish hospital study ${ }^{(21)}$

Impact of pharmacy-led medication reconciliation on medication errors during transition in the hospital setting ${ }^{(19)}$
Redução da carga horária da equipe de enfermagem e implantação de programas de educação permanente para a equipe de saúde.

Um sistema CPOE (Computerized Physician Order Entry) associado à verificação do farmacêutico clínico.

Um modelo multivariado para prever possíveis eventos adversos potenciais a medicamentos como estratégia para melhorar a alocação do farmacêutico clínico e subsequente segurança do paciente.

Uma ferramenta de apoio à decisão clínica incorporada no sistema CPOE (Computerized Physician Order Entry).

Um Sistema Automatizado de Medicação - AMS integra registros eletrônicos de administração (eMARs), dispensação automática assistida por código de barras (BCMA).

Envolvimento do farmacêutico clínico no processo de reconciliação de medicamentos na internação hospitalar 


\begin{tabular}{|l|l|}
\hline $\begin{array}{l}\text { Pharmacist-assisted electronic prescribing } \\
\text { at the time of admission to an inpatient } \\
\text { orthopaedic unit and its impact on medication } \\
\text { errors: a pre- and post intervention study(27) }\end{array}$ & $\begin{array}{l}\text { Envolvimento do farmacêutico clínico no processo de } \\
\text { reconciliação de medicamentos na internação e durante } \\
\text { a prescrição eletrônica com uso do eMMS (sistema } \\
\text { eletrônico de gerenciamento de medicamentos) em } \\
\text { pacientes ortopédicos hospitalizados. }\end{array}$ \\
\hline $\begin{array}{l}\text { Experiences with Lean Six Sigma as } \\
\text { improvement strategy to reduce parenteral } \\
\text { medication administration errors and } \\
\text { associated potential risk of harm }\end{array}$ & $\begin{array}{l}\text { Programa de melhoria hospitalar, Lean Six Sigma. } \\
\text { Intervenção personalizada para os problemas: ciclo } \\
\text { de cinco etapas, DMAIC (Definir, Medir, Analisar, } \\
\text { Melhorar, Controlar), com treinamentos da equipe, } \\
\text { alterações de rotinas no processo de administração de } \\
\text { medicamentos, cartazes e folders. }\end{array}$ \\
\hline $\begin{array}{l}\text { Implementing technologies to prevent } \\
\text { medication errors at a high-complexity } \\
\text { hospital: analysis of cost and results (28) }\end{array}$ & $\begin{array}{l}\text { Integração de várias tecnologias na cadeia } \\
\text { medicamentosa na prevenção dos erros de medicação. }\end{array}$ \\
\hline $\begin{array}{l}\text { Risk factors for medication errors in the } \\
\text { electronic and manual prescription (20) }\end{array}$ & $\begin{array}{l}\text { Prescrição eletrônica (PE) com suporte à decisão clínica } \\
\text { X prescrição manual (PM), identificando os fatores de } \\
\text { risco para ocorrência de erros de medicação. }\end{array}$ \\
\hline
\end{tabular}

Fonte: Autores (2020).

A presente revisão integrativa permitiu a identificação de estratégias para redução de erros de medicação durante a hospitalização do paciente adulto. Houve uma grande variabilidade entre os países dos estudos analisados e sintetizados, mostrando a importância e preocupação que a temática representa e que há um grande interesse das instituições hospitalares em mitigar a ocorrência de erros de medicação.

Ressalta-se o envolvimento do farmacêutico nas atividades clínicas diárias nas unidades de internação, desde a admissão até a alta do paciente, atuando no processo de reconciliação medicamentosa na internação hospitalar, acompanhando e intervindo em todo o processo de administração de medicamentos. As atividades desenvolvidas pelo farmacêutico na clínica são fundamentais para o uso racional de fármacos, contribuindo para o alcance de resultados terapêuticos previstos, com a minimização de riscos de erros de medicação(18-19,22,24,27).

É importante lembrar que o relatório intitulado "Errar é humano: construindo um sistema de saúde mais seguro", do Comitê de Qualidade de Assistência à Saúde na América do Instituto de Medicina (EUA), em 1999, descreveu os erros humanos no sistema de saúde e destacou a importância do profissional farmacêutico na reconciliação medicamentosa e prescrição(27).

Outros estudos também revelam o impacto significativo da intervenção do farmacêutico no uso de sistemas informatizados de prescrição eletrônica (Computerized Physician Order Entry - CPOE), e de gerenciamento de medicamentos (Electronic Medication Management System - EMMS). Com o apoio dessas ferramentas tecnológicas, observaram-se melhoras no desfecho clínico do paciente devido à precisão do tratamento medicamentoso, melhoria na segurança do paciente com a prevenção de eventos adversos e, consequentemente, economia de custos ${ }^{(19,27)}$.

A implantação e/ou melhorias de tecnologias da informação foram as estratégias mais encontradas nos estudos avaliados, mostrando redução nas taxas de erros de medicação, facilitando a comunicação interdisciplinar e reduzindo atrasos na correção de prescriçôes ${ }^{(18,20-22,26-28)}$. 
Houve estudos em que o sistema informatizado de prescrição eletrônica (CPOE) foi incorporado a módulos de apoio à decisão clínica e ao sistema eletrônico de gerenciamento de medicamentos (EMMS). Estas ferramentas de apoio favoreceram a integração de informações farmacêuticas compreensíveis e confiáveis, possibilitaram acesso aos dados sobre eventuais interações medicamentosas ou efeitos adversos, tornando os profissionais habilitados para administrar medicamentos de forma segura e precisa. Os autores dos estudos indicam o uso das ferramentas tecnológicas para a obtenção de resultados satisfatórios para a segurança do paciente, tanto na redução de medicações incorretas como de eventos adversos a medicamentos ${ }^{(26-27)}$.

Acredita-se que o CPOE favorece a leitura e a compreensão da prescrição, reduzindo sua ilegibilidade e eliminando rasuras. O CPOE focaliza informações fundamentais à prescrição (via, diluição, dose, frequência), nome do princípio ativo e prescrições completas. Em um dos estudos, foi verificada a diminuição de ocorrências de alergias. Emm contraponto, houve aumento de fatores de risco como "falta de checagem" e "falta de identificação do número de inscrição no conselho profissional do prescritor". É de se reconhecer, entretanto, que quando a informatização é bem utilizada, promove facilidade e precisão nos registros dos profissionais da equipe de saúde, especialmente médicos e enfermeiros, aumentando a segurança dos pacientes e reduzindo fatores de risco para tais situações. Assim sendo, 0 CPOE, por si só, não elimina a possibilidade de erros de medicação ${ }^{(20)}$.

A educação permanente foi elencada como estratégia nos estudos selecionados $(17,22-23,25,28)$; quando conduzida como uma prática constante, favorece a prevenção de erros e óferece segurança ao profissional(25).

Estratégias educacionais mediadas por simulação, jogos e outros demonstram ser importantes para melhoria da comunicação multidisciplinar, desenvolvimento da cultura de segurança, integração ao uso de ferramentas tecnológicas e, também, para redução das taxas de incidentes envolvendo medicações ${ }^{(17,22)}$. Considerada uma estratégia de baixo custo, sugere-se que deva ser cada vez mais explorada e valorizada pelos gestores, de modo a prevenir erros na administração de medicamentos e garantir a segurança do paciente.

Entre os estudos analisados, a redução da carga de trabalho e a constituição suficiente da equipe de enfermagem foram relatadas como estratégias na redução de erros de medicação ${ }^{(17,22-23)}$. Em um estudo realizado em unidade de terapia intensiva, foi identificado que a carga de trabalho pesada devido ao grande número de pacientes, somada à fadiga, sono e estresse, agravaram a ocorrência de diferentes erros no processo de administração de medicamentos ${ }^{(23)}$.

O número adequado de profissionais é indispensável para o cuidado seguro, sendo responsabilidade dos gestores e instituição proverem condições favoráveis de trabalho aos recursos humanos nas unidades. A adequação da carga horária e o quantitativo de profissionais, segundo o número e necessidades dos pacientes, representam um importante papel na redução de erros de medicação, como também menor incidência de agravos à saúde dos trabalhadores ${ }^{(22-23)}$.

Programas de qualidade hospitalar foram elencados como estratégia de prevenção de erros de medicação nos estudos ${ }^{(17,25)}$. Alguns programas de intervenção com foco em erros de medicação incluem medidas organizacionais, de tecnologia da informação, educacionais e baseadas em otimização de processos. Programas como o da Joint Commission International (JCl) e o método Lean Six Sigma são desenvolvidos em instituições hospitalares, e podem intensificar a qualidade dos sistemas de saúde, melhorar o clima organizacional, satisfação, segurança e conhecimento dos profissionais e, consequentemente, aumentam sua capacidade e motivação para prevenir erros de medicação e alcançar práticas bemsucedidas de melhoria de qualidade do cuidado.

Há de se ressaltar a importância desse tema na formação do enfermeiro, de modo que esforços sejam empreendidos para que os futuros profissionais cheguem ao mercado 
de trabalho já preparados para garantir a qualidade dos sistemas de saúde, especialmente no tocante à capacidade de prevenir erros de medicação. Investimentos acadêmicos têm sido reportados na literatura com roteiros para avaliar a segurança na administração de medicamentos por estudantes de enfermagem ${ }^{(29)}$, assim como o envolvimento e participação do paciente como interessado em atentar para a própria segurança na recepção de cuidados ${ }^{(30)}$. À medida que passamos a contar com profissionais mais preparados e com o aumento da literacia em saúde, teremos condições mais favoráveis para que as organizações de saúde obtenham êxito em seus programas de qualidade e segurança do paciente.

\section{CONCLUSÃO}

Constatou-se que as tecnologias da comunicação na assistência à saúde se tornaram ferramentas importantes à equipe multiprofissional, aumentando a qualidade da assistência prestada ou incrementando a confiabilidade das intervenções necessárias.

Associadas às tecnologias, o envolvimento do farmacêutico nas atividades diárias nas unidades de internação com a equipe médica e de enfermagem resultará em melhorias na redução dos eventos adversos relacionados a medicamentos e na qualidade da assistência.

Aliam-se a essas duas estratégias o desenvolvimento de programas de educação continuada à equipe multiprofissional focados em qualidade, bem como a postura e compromisso da gestão hospitalar para com as condições de trabalho de seu pessoal, o capital mais importante da instituição que administra; a redução da carga de trabalho e o contingente suficiente da equipe de enfermagem não podem deixar de ser consideradas quando se busca compor e eleger estratégias de redução de erros de medicação.

A análise dos estudos selecionados evidencia a necessidade de se buscar conhecer continuamente resultados de estudos empreendidos em prol da garantia de oferecimento de segurança ao paciente e eliminação de falhas no processo de administração de medicamentos. As estratégias elencadas nos estudos complementam-se e demonstram a importância de pesquisas multidisciplinares nesta temática.

\section{REFERÊNCIAS}

1. Carlesi KC, Padilha KG, Toffoletto MC, Henriquez-Roldán C, Juan MAC. Patient Safety Incidents and Nursing Workload. Rev Latino-Am Enfermagem [Internet]. 2017 [acesso em 10 dez 2020]; 25. Disponível em: https://doi.org/10.1590/1518-8345.1280.2841.

2. Dirik HF, Samur M, Intepeler SS, Hewison A. Nurses' identification and reporting of medication errors. J Clin Nurs. [Internet]. 2019 [acesso em 10 dez 2020]; 28(5-6): 931-8. Disponível em: https://doi. org/10.1111/jocn.14716.

3. Riaz MK, Riaz M, Latif A. Medication errors and strategies for their prevention. Pak. J. Pharm. Sci. [Internet]. 2017 [acesso em $10 \mathrm{dez}$ 2020]; 30(3): 921-8. Disponível em: http://www.pjps.pk/wp-content/ uploads/pdfs/30/3/Paper-33.pdf.

4. Farzi S, Irajpour A, Saghaei M, Ravaghi H. Causes of medication errors in intensive care units from the perspective of healthcare professionals. J Res Pharm Pract. [Internet]. 2017 [acesso em 11 dez 2020]; 6(3).

Disponível em: https://doi.org/10.4103/jrpp.JRPP 174.

5. World Health Organization (WHO). [Internet]. WHO Global Patient Safety Challenge: Medication Without Harm. Geneva: WHO; 2017 [acesso em 11 dez 2020]. Disponível em: https://www.who.

int/initiatives/medication-without-harm\#: :text=Medication\%20Without\%20Harm\%20aims\%20 
6. Medication Without Harm: WHO's Third Global Patient Safety Challenge. The lancet. [Internet]. 2017 [acesso em $11 \mathrm{dez}$ 2020]; 389. Disponível em: https://www.thelancet.com/pdfs/journals/lancet/PIIS01406736(17)31047-4.pdf.

7. Johnson A, Guirguis E, Grace Y. Preventing medication errors in transitions of care: a patient case approach. J Am Pharm Assoc. [Internet]. 2015 [acesso em 11 dez 2020]; 55(2). Disponível em: https:// www.sciencedirect.com/science/article/abs/pii/S1544319115300492?via\%3Dihub.

8. Zaree TY, Nazari J, Jafarabadi MA, Alinia T. Impact of psychosocial factors on occurrence of medication errors among tehran public hospitals nurses by evaluating the balance between effort and reward. Saf Health Work [Internet]. 2018 [acesso em 11 dez 2020]; 9(4): 447-53. Disponível em: https://doi. org/10.1016/j.shaw.2017.12.005.

9. National Coordinating Council for Medication Error Reporting and Prevention (NCCMERP). Taxonomy of medication errors [Internet]. 2016 [acesso em $12 \mathrm{dez}$ 2020]. Disponível em: http://www.nccmerp.org/ sites/default/files/taxonomy2001-07-31.pdf.

10. Nuckols TK, Smith-Spangler C, Mortoon SC, Asch SM, Patel VM, Anderson LJ, et al. The effectiveness of computerized order entry at reducing preventable adverse drug events and medication errors in hospital settings: a systematic review and meta-analysis. Systematic Reviews [Internet]. 2014 [acesso em 12 dez 2020]; 3. Disponível em: https://doi.org/10.1186/2046-4053-3-56.

11. Elliott M, Liu Y. The nine rights of medication administration: an overview. Br J Nurs. [Internet]. 2010 [acesso em 12 dez 2020]; 19(5): 300-5. Disponível em: https://doi.org/10.12968/bjon.2010.19.5.47064.

12. Ministério da Saúde (BR). Agencia Nacional de Vigilância Sanitária. Anexo 3: protocolo de segurança na prescrição, uso e administração de medicamentos [Internet]. Brasília: Ministério da Saúde; 2013 [acesso em 05 abr 2021]. Disponível em: https://portaldeboaspraticas.iff.fiocruz.br/wp-content/ uploads/2020/01/protoc identificacaoPaciente.pdf.

13. Whittemore R, Knafl K. The integrative review: updated methodology. J. Adv. Nurs. [Internet]. 2005 [acesso em 14 dez 2020]; 52(5): 546-53. Disponível em: https://dx.doi.org/10.1111/j.13652648.2005.03621.x.

14. Santos CM da C, Pimenta CA de M, Nobre MRC. The PICO strategy for the research question construction and evidence search. Rev Latino-Am Enferm [Internet]. 2007 [acesso em 14 dez 2020]; 15(3): 508-11. Disponível em: https://dx.doi.org/10.1590/S0104-11692007000300023.

15. Ursi ES, Galvão CM. Prevenção de lesões de pele no perioperatório: revisão integrativa da literatura. Rev. Latino-Am. Enfermagem [Internet]. 2006 [acesso em 14 dez 2020]; 14 (1): 124-31. Disponível em: https://doi.org/10.1590/S0104-11692006000100017.

16. Melnyk BM, Fineout-Overholt E. Evidence based practice in nursing \& healthcare: a guide to best practice. 2. ed. Philadelphia: Lippincott Williams \& Wilkins; 2011.

17. Wang $H$, Jin J, Feng $X$, Huang $X$, Zhu L, Zhao $X$, et al. Quality improvements in decreasing medication administration errors made by nursing staff in an academic medical center hospital: a trend analysis during the journey to Joint Commission International accreditation and in the post-accreditation era. Ther Clin Risk Manag. [Internet]. 2015 [acesso em 30 nov 2020]; 5(11): 393-406. Disponível em: https://doi. org/10.2147/TCRM.S79238.

18. Hernandez F, Majoul E, Montes-Palacios C, Antignac M, Cherrier B, Doursounian L, et al. An Observational Study of the Impact of a Computerized Physician Order Entry System on the Rate of Medication Errors in an Orthopaedic Surgery Unit. PLoS ONE [Internet]. 2015 [acesso em 26 nov 2020]; 10(7). Disponível em: https://doi.org/10.1371/journal.pone.0134101.

19. Smith L, Mosley J, Lott S, Cyr Jr. E, Amin R, Everton E, et al. Impact of pharmacy-led medication reconciliation on medication errors during transition in the hospital setting. Pharmacy Pract. [Internet]. 2015 [acesso em 30 nov 2020]; 13(4). Disponível em: http://dx.doi.org/10.18549/PharmPract.2015.04.634. 
20. Volpe CRG, Melo EMM de, Aguiar LB de, Pinho DLM, Stival MM. Risk factors for medication errors in the electronic and manual prescription. Rev. Latino-Am. Enfermagem [Internet]. 2016 [acesso em 29 nov 2020]; 24. Disponível em: http://dx.doi.org/10.1590/1518-8345.0642.2742.

21. Risor BW, Lisby M, Sorensen J. An automated medication system reduces errors in the medication administration process: results from a Danish hospital study. Eur J Hosp Pharm. [Internet]. 2016 [acesso em 27 nov 2020]; 23 (4): 189-96. Disponível em: https://doi.org/10.1136/ejhpharm-2015-000749.

22. Farzi S, Irajpour A, Saghaei M, Ravaghi H. Causes of Medication Errors in Intensive Care Units from the Perspective of Healthcare Professionals. J Res Pharm Pract [Internet]. 2017 [acesso em 26 nov 2020]; 6(3): 158-65. Disponível em: https://doi.org/10.4103/jrpp.JRPP 1747.

23. Fathi A, Hajizadeh M, Moradi K, Zandian H, Dezhkameh M, Kazemzadeh S, et al. Medication errors among nurses in teaching hospitals in the west of Iran: what we need to know about prevalence, types, and barriers to reporting. Epidemiol Health [Internet]. 2017 [acesso em 26 nov 2020]; 17(39). Disponível em: https://doi.org/10.4178/epih.e2017022.

24. Nguyen Tri-Long, Leguelinel-Blache G, Kinowski Jean-Marie, Roux-Marson C, Rougier M, Spence $\mathrm{J}$, et al. Improving medication safety: Development and impact of a multivariate model-based strategy to target high-risk patients. PLoS ONE [Internet]. 2017 [acesso em 27 nov 2020]; 12(2). Disponível em: https://doi.org/10.1371/journal.pone.0171995.

25. Plas AV de, Slikkerveer M, Hoen S, Schrijnemakers R, Driessen J, Vries F de, et al. Experiences with Lean Six Sigma as improvement strategy to reduce parenteral medication administration errors and associated potential risk of harm. BMJ Qual Improv Rep. [Internet]. 2017 [acesso em 28 nov 2020]; 6(1). Disponível em: https://doi.org/10.1136/bmjquality.u215011.w5936.

26. Pouliot JD, Neal EB, Lobo BL, Hargrove F, Gupta RK. The Role of Computerized Clinical Decision Support in Reducing Inappropriate Medication Administration During Epidural Therapy. Hospital Pharmacy [Internet]. 2018 [acesso em 27 nov 2020]; 53(3):170-6. Disponível em: https://doi. org/10.1177/0018578717741392.

27. Tran T, Taylor SE, Hardidge A, Mitri E, Aminian P, George J, et al. Pharmacist-assisted electronic prescribing at the time of admission to an inpatient orthopaedic unit and its impact on medication errors: a pre- and post intervention study. The Adv Drug Saf. [Internet]. 2019 [acesso em 28 nov 2020]; 10. Disponível em: https://doi.org/10.1177/2042098619863985.

28. Vilela RPB, Jericó M de C. Implementing technologies to prevent medication errors at a highcomplexity hospital: analysis of cost and results. Einstein (São Paulo) [Internet]. 2019 [acesso em 29 nov 2020]; 17(4). Disponível em: https://doi.org/10.31744/einstein journal/2019gs4621.

29. Cancino KD, Arias M, Caballero E, Escudero E. Development of a safe drug administration assessment instrument for nursing students. Rev. Latino-Am. Enfermagem. 2020 [acesso em 16 jan 2021]; 28. Disponível em: http://dx.doi.org/10.1590/1518-8345.2989.3246.

30. Costa DG da, Moura GMSS de, Pasin SS, Costa FG da, Magalhães AMM de. Patient experience in coproduction of care: perceptions about patient safety protocols. Rev. Latino-Am. Enfermagem. [Internet]. 2020 [acesso em 16 jan 2021]; 28. Disponível em: https://doi.org/10.1590/1518-8345.3352.3272. 


\section{ESTRATÉGIAS PARA A REDUÇÃO DE ERROS DE MEDICAÇÃO DURANTE A HOSPITALIZAÇÃO: REVISÃO INTEGRATIVA}

\section{RESUMO:}

Objetivo: sintetizar o conhecimento relacionado às estratégias para redução de erros de medicação durante a hospitalização de pacientes adultos. Método: revisão integrativa da literatura realizada em outubro de 2020 por meio da busca de publicações nas bases de dados eletrônicas: Web of Science, Pubmed, Cumulative Index of Nursing and Allied Health Literature, Literatura Latino-Americana e do Caribe em Ciências da Saúde e Google Scholar. Resultados: amostra composta por 12 estudos, cujas estratégias para a redução de erros de medicação foram apresentadas de acordo com as categorias: envolvimento do farmacêutico clínico nas atividades clínicas, implantação de tecnologias da informação, estratégias educacionais mediadas por simulação e jogos, e redução da carga de trabalho. Conclusão: associado às tecnologias, o envolvimento do farmacêutico clínico com a equipe médica e de enfermagem resultará em melhorias na redução dos eventos adversos a medicamentos e na qualidade da assistência prestada ao paciente. DESCRITORES: Erros de Medicação; Segurança do Paciente; Sistemas de Medicação no Hospital; Gestão da Segurança; Cuidados de Enfermagem.

\section{ESTRATEGIAS PARA LA REDUCCIÓN DE ERRORES DE MEDICACIÓN DURANTE LA HOSPITALIZACIÓN: REVISIÓN INTEGRADORA}

\section{RESUMEN:}

Objetivo: sintetizar el conocimiento relacionado a las estrategias para reducción de errores de medicación durante la hospitalización de pacientes adultos. Método: revisión integradora de la literatura realizada en octubre de 2020 por medio de la búsqueda de publicaciones en las bases de datos electrónicas: Web of Science, Pubmed, Cumulative Index of Nursing and Allied Health Literature, Literatura Latinoamericana y del Caribe en Ciencias de la Salud y Google Scholar. Resultados: muestra compuesta por 12 estudios, cuyas estrategias para reducir los errores de medicación se presentaron según las categorías: implicación del farmacéutico clínico en las actividades clínicas, implementación de tecnologías de la información, estrategias educativas mediadas por simulación y juegos, y reducción de la carga de trabajo. Conclusión: asociada a las tecnologías, la implicación del farmacéutico clínico con el equipo médico y de enfermería se traducirá en mejoras en la reducción de los efectos adversos de los medicamentos y en la calidad de la atención al paciente.

DESCRIPTORES: Errores de Medicación; Seguridad del Paciente; Sistemas de Medicación en el Hospital; Gestión da Seguridad; Cuidados de Enfermería.

Recebido em: 17/02/2021

Aprovado em: 03/08/2021

Editora associada: Luciana Alcântara Nogueira

Autor Correspondente:

Isabel Amélia Costa Mendes

Universidade de São Paulo - Ribeirão Preto, SP, Brasil

E-mail: iamendes@usp.br

Contribuição dos autores:

Contribuições substanciais para a concepção ou desenho do estudo; ou a aquisição, análise ou interpretação de dados do estudo - Costa CR de B, Santos SS dos, Godoy S de, Alves LMM, Silva IR, Mendes IAC; Elaboração e revisão crítica do conteúdo intelectual do estudo - Costa CR de B, Godoy S de, Alves LMM, Silva IR, Mendes IAC; Responsável por todos os aspectos do estudo, assegurando as questões de precisão ou integridade de qualquer parte do estudo - Costa CR de B, Santos SS dos, Godoy S de, Alves LMM, Mendes IAC. Todos os autores aprovaram a versão final do texto.

Copyright (? 2021 Este é um artigo em acesso aberto distribuído nos termos da Licença Creative Commons Atribuição, que permite o uso irrestrito, a distribuição e reprodução em qualquer meio desde que o artigo original seja devidamente citado. 